\title{
Editorial
}

\section{Lasers in the Preservation of Cultural Heritage}

\author{
Costas Fotakis, ${ }^{1}$ Wolfgang Kautek, ${ }^{2}$ and Marta Castillejo ${ }^{3}$ \\ ${ }^{1}$ Foundation for Research and Technology-Hellas, Institute of Electronic Structure and Laser, 71110 Heraklion, Greece \\ ${ }^{2}$ Department of Physical Chemistry, University of Vienna, 1090 Vienna, Austria \\ ${ }^{3}$ Spanish National Research Council (CSIC), Serrano 117, 28006 Madrid, Spain
}

Received 31 December 2006; Accepted 31 December 2006

Copyright (C) 2006 Costas Fotakis et al. This is an open access article distributed under the Creative Commons Attribution License, which permits unrestricted use, distribution, and reproduction in any medium, provided the original work is properly cited.

To date, it has been proven that modern laser technology may well serve the future of our past, the preservation and revelation of cultural heritage. This field is now coming to an era of maturity both in terms of the level of understanding of the fundamental aspects involved and the practical diagnostics and restoration applications, which have been demonstrated. The workshop on "Lasers in the Preservation of Cultural Heritage," held in June 27-28, 2006 in St. Petersburg, Russia, was organized in the framework of the Laser Optics Conference, attracting contributions which reflect the state of the art and the potential for future developments. The workshop took place in the premises of the State Heritage Museum and included presentations of fundamentals and test case studies, some of which are described in this special issue of laser chemistry.

In particular, laser-based spectroscopic techniques of noninvasive character, such as laser-induced fluorescence (LIF) and raman spectroscopy are now capable of providing in situ and remote analytical information of organic materials. Novel aspects of laser-induced breakdown spectroscopy (LIBS) for the elemental analysis of the original materials or pollutants of artifacts by using ultra-fast lasers (fs-LIBS) were also presented, together with a range of test case applications.

Besides the established use of lasers emitting pulses of nanosecond duration for cleaning applications, femtosecond lasers also present interesting new possibilities for demanding conservation cases (e.g., for cleaning of polychromes and sensitive materials like those used in modern paintings). It is the extremely high spatial resolution of the ablation processes and the minimal thermal and photochemical effects which may be achieved by using femtosecond lasers that leads to the success of these applications.

Finally, interferometric techniques for the detection and evaluation of defects and the structural status of artifacts appear to come to age. Along these lines, optical coherence to- mography (OCT) was reported to be a promising technique for exciting new applications.

Costas Fotakis Wolfgang Kautek Marta Castillejo 